\title{
Evaluation of High Performance Rotor Cooling Techniques for Permanent Magnet Electric Motors
}

\author{
Jasper Nonneman*†, Brent Van der Sijpe*, Ilya T’Jollyn*†, Steven Vanhee ${ }^{\ddagger}$, Joachim Druant ${ }^{\ddagger}$, Michel De Paepe*† \\ Email: \{Jasper.Nonneman,Brent.VanderSijpe,Ilya.TJollyn,Michel.DePaepe\}@UGent.be \{Steven.Vanhee,Joachim.Druant\}@Dana.com \\ *Department of Electromechanical, Systems and Metal Engineering, Ghent University, Sint-Pietersnieuwstraat 41, 9000 Ghent, Belgium \\ †Flanders Make@UGent - Core lab EEDT-MP, Belgium \\ ‡Dana Belgium NV, Ten Briele 3, 8200 Brugge, Belgium
}

\begin{abstract}
A thermal analysis of an interior permanent magnet machine using a lumped parameter model is presented, with the objective to evaluate several rotor cooling techniques for electric vehicle applications which are needed in order to achieve higher power densities. Two of the selected rotor cooling techniques involve cooling of the shaft by pumping a liquid through a channel in the shaft and by implementing a rotating heat pipe. The third selected rotor cooling technique is accomplished by pointing liquid jets towards the hub of the rotor. The lumped parameter model and implementation of these newly modeled cooling techniques are described. From the temperature distributions of the electrical machine resulting from water jacket cooling in combination with the different rotor cooling techniques, it was revealed that when the rotor of an electrical motor contains a hub, shaft cooling methods are ineffective. Rotor jet cooling however resulted in the most effective cooling approach to minimize permanent magnet temperatures.
\end{abstract}

Keywords-Electric motor cooling, Lumped parameter model, Thermal management, Rotor cooling, Permanent magnet synchronous motor

\section{INTRODUCTION}

Due to global warming and the increasingly stringent legislation about emissions of vehicles, there is a strong tendency to shift to hybrid or all-electrical drivetrains in the automotive industry. The Interior Permanent Magnet Synchronous Motor (IPMSM) is the most popular electrical motor for electrical vehicle applications [1]. Due to increasing power densities, it becomes more and more challenging to keep the IPMSM at acceptable temperature levels. Especially the winding and permanent magnet (PM) temperatures are the major constraints, as these should be kept in their operational temperature ranges. Excessive winding temperatures cause deterioration of the conductors and accelerated insulation aging [2]. For traction applications, depending on the insulation class, maximum coil temperatures are approximately $180^{\circ} \mathrm{C}$. The permanent magnets are susceptible to irreversible demagnetization at high temperatures. A conventional stator cooling technique is liquid jacket cooling, while more advanced techniques are end winding cooling [3] [4] and direct slot cooling [5] [6]. For high-power density electrical machines where the maximum rotor temperature is limited as the permanent magnets are located in the rotor, these cooling approaches often do not provide the desired cooling performance, resulting in a need for innovative, high performance rotor cooling techniques.

As the motor performance is highly dependent on the temperature distribution in the electrical machine, accurate thermal models are needed to design and improve these machines. In this work, the electrical machine will be modeled using an advanced lumped parameter model (LPM) [7]. A flowchart of the model is shown in Fig. 2. The model assumes axial symmetry between the different stator/rotor poles and as such models only half of a stator and rotor tooth as shown in Fig. 1. Within the axial direction and at the end plates, the geometry of the motor is relatively simple and low thermal gradients occur due to the relatively high thermal conductivity of the components. Therefore an analytical approach can be used for the equivalent thermal networks of the nodes. A difference is made between nodes without internal heat generation and with internal heat generation. The equivalent networks are shown in Fig. 2, where $\mathrm{R}$ is the thermal resistance, $\mathrm{C}$ the heat capacity and $\mathrm{Q}$ the heat generation. It is more difficult to apply this lumped parameter technique in the radial and tangential direction, because high and 2D temperature gradients occur due to low thermal conductivities and interface resistances. The proposed solution is to use a 2D FE model to accurately determine the temperature distribution in these directions and to extract a 2D LPM from this model. Therefore a coarse discretization is put on top of the different components of the 2D geometry and the amount of coarse nodes is increased for components with a high temperature gradient. The cells resulting from this coarse discretization serve as a basis for the different thermal nodes of the 2D LPM and the thermal resistances are extracted by the average temperature difference between the two neighboring cells divided by the heat transfer between them.

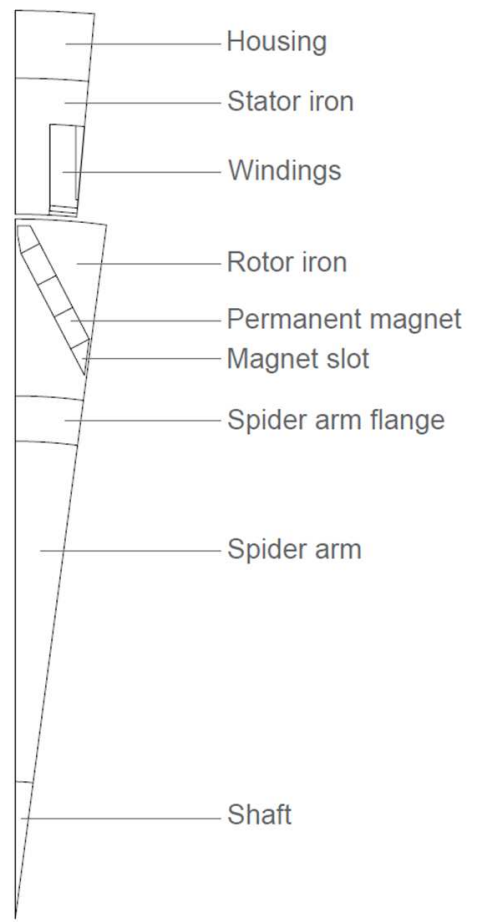

Fig. 1: Simplified cross-section of the IPMSM 

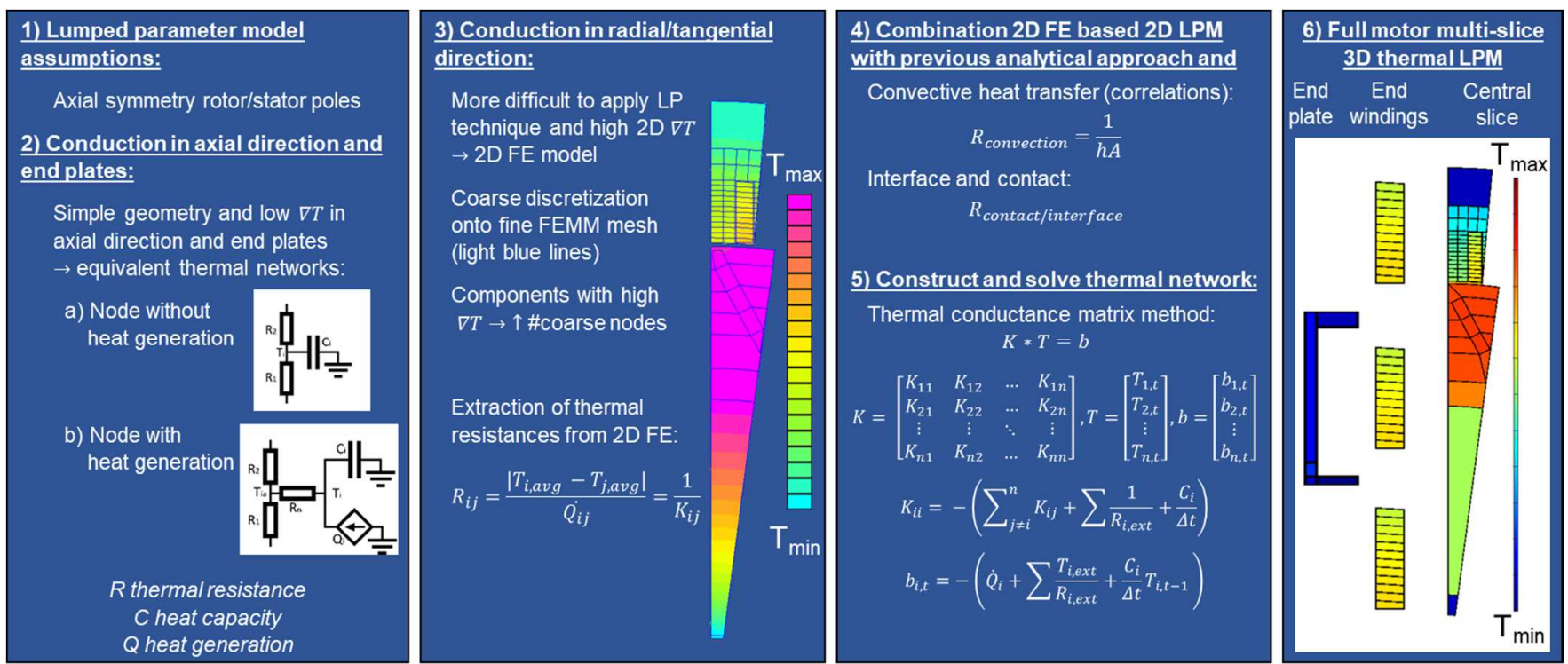

Fig. 2: 3D LP model flowchart (i, j and $\mathrm{n}$ represent node indices; $R_{i j}$ thermal resistance, $Q_{i j}$ the heat flow and $K_{i j}$ thermal conductance between these nodes; $T_{\text {avg }}$ average node temperature in FEMM; h convection coefficient; A heat transfer area; $K$ thermal conductance matrix; T array of node temperatures; $b$ dependent on heat input, heat transfer to external media and temperature at previous time iteration t; $\dot{Q}_{l}$ heat coming into node i; $R_{i, \text { ext }}$ thermal resistance to the external temperature $T_{i, \text { ext }}$ of the medium; $C_{i}$ heat capacity of node $\mathrm{i}$ and $\Delta t$ time step)

The combination of the 2D FE based 2D LPM with the analytical approach and the thermal resistances by convection calculated from correlations and interface and contact resistances, results in a full $3 \mathrm{D}$ thermal LPM for the electric machine. The machine can also be split into two or more slices in the axial direction, resulting in a multi-slice 3D LPM with which also the maximum temperature in the axial direction can be determined. The thermal conductance matrix method is used to construct and solve the whole thermal network, where the discretized heat balance between the nodes of the coarse discretization is written in matrix formulation with the equations as shown in Fig. 2. The result is a full motor multislice 3D LPM, which can be used to simulate the temperature distribution within the motor based on the coarse discretization of the components.

The considered IPMSM is designed to deliver a continuous power of $130 \mathrm{~kW}$ at $2400 \mathrm{rpm}$. To verify the effectiveness of the selected rotor cooling techniques, an operating point with relatively high losses in the rotor part was chosen. This operating point is at high speed $(\omega=6000 \mathrm{rpm})$ and low torque $(\mathrm{T}=160 \mathrm{Nm})$. Due to high frequencies of the magnetic field, the high angular velocity results in high iron losses in the stator $\left(\mathrm{P}_{\text {stator }}\right)$ and rotor $\left(\mathrm{P}_{\text {rotor }}\right)$ and high permanent magnet losses $\left(\mathrm{P}_{\mathrm{PM}}\right)$. Another consequence of the high speed is high friction with the air inside the machine $\left(\mathrm{P}_{\text {windage }}\right)$ and in the bearings $\left(\mathrm{P}_{\text {friction }}\right)$. Due to low torque, relatively low copper losses $\left(\mathrm{P}_{\text {copper }}\right)$ are seen. An overview of the losses is shown in TABLE I.

TABLE I: MACHINE LOSSES

\begin{tabular}{|c|c|}
\hline Location & Losses $(\mathbf{W})$ \\
\hline$P_{\text {copper }}$ & 1880 \\
\hline$P_{\text {stator }}$ & 2154 \\
\hline$P_{\text {rotor }}$ & 239 \\
\hline$P_{\text {windage }}$ & 100 \\
\hline$P_{P M}$ & 398.1 \\
\hline$P_{\text {friction }}$ & 300 \\
\hline
\end{tabular}

Further in this paper, firstly the cooling methods and implementation in the lumped parameter model are described. Afterwards, the simulated thermal performance of the different methods with the LPM will be discussed.

\section{ROTOR COOLING METHODS}

In the following subsections, the selected rotor cooling techniques will be explained based on their working principles.

In a hollow shaft liquid cooling (HSLC) setup, coolant is forced through an axial hole in the shaft with the purpose of cooling the rotor [6] [8]. This coolant can be a gas or a liquid. In the following, the focus is on liquid coolants, as cooling of high power density motors is the aim of this work. The most conventional type of a hollow shaft cooled electrical machine uses two rotational couplings to connect the hollow space in the shaft with an external cooling circuit. The HSLC working principle is seen in Fig. 3, together with the general geometry of the IPMSM. Note that the rotor iron is carried by a steel structure, referred to as the spider arm. The heat transfer from shaft to the coolant is characterized by forced convection within a rotating channel. Due to the heat transfer near the wall, an annulus is formed with elevated temperature. This means that the liquid near the wall decreases in density while the colder core liquid has a higher density. By means of the centrifugal and Coriolis force, this colder core coolant is forced towards the wall, creating secondary flows and enhancing the mixing of the overall flow, resulting in a smaller boundary layer thickness and hence a higher heat transfer rate. In general, the heat transfer is improved by increasing rotational speed and is only little dependent on the mass flow rate.

A rotating heat pipe (RHP) is a passive, two-phase device capable of transferring large amounts of heat between its outer ends [9]. Its working principle primary relies on phase transition of the working fluid. As shown in Fig. 4, heat addition causes the fluid to vaporize in the evaporator. The vapor is driven towards the condenser side of the rotary heat 
pipe by a small pressure difference, where it condenses to liquid again. This pressure difference in the vapor phase results in a small temperature difference over the RHP. As the inner surface of the RHP is slightly conical and the smallest diameter occurs at the condenser side, a taper angle is formed between both sides. As the sealed container is rotating about the longitudinal axis, the condensate is carried towards the evaporator side by the work of a component of the centrifugal force and the cycle continues. Hence, the centrifugal force acting on the fluid is the driving force of the superior performance of RHPs compared to stationary heat pipes which rely on capillary forces. In case of shaft cooling methods, the spider arm is mostly the bottleneck in the heat transfer path from rotor iron and magnets to the coolant, due to the small thickness and moderate thermal conductivity of steel resulting in a rather high overall thermal resistance. To reduce the thermal resistance of the spider arm in case of the shaft cooling methods, potting material $\left(\mathrm{k}_{\text {potting }}=2.3 \mathrm{~W} / \mathrm{mK}\right)$ is inserted in the space between the spider arm and the shaft, as indicated in Fig. 3.

A final rotor cooling method that is evaluated in this study is rotor jet cooling (RJC) with the aim to decrease permanent magnet temperatures in PMSMs [10] [11]. The concept of this rotor cooling technique is to point a jet on the inside of the rotor of the electrical machine. The liquid jet can be realized by implementing a hollow shaft arrangement equipped with nozzles or positioning nozzles in the end flanges (see Fig. 5). When spraying the liquid on the inner wall of the rotor, large heat transfer coefficients occur in the jet impingement region. Additionally, a thin film is formed due to the rotational speed of the rotor, which further enhances the heat transfer. By inserting a small slope on the inner wall of the rotor, the liquid will flow to a larger radial position where appropriate output channels should be implemented. Since the fluid is in direct contact with the electromagnetic materials, a dielectric coolant such as oil should be used.

\section{THERMAL LUMPED PARAMETER MODEL}

To evaluate the rotor cooling methods described in the previous section, an existing 3D LPM [7] is extended with the proposed methods. For hollow shaft liquid cooling, specialized heat transfer correlations are proposed by SeghirOuali et al. [12]. The Nusselt number is a function of axial Reynolds number $\mathrm{Re}_{\mathrm{a}}=4 \mathrm{~V} \rho /(\pi \mu \mathrm{D})$ and rotational Reynolds number $\operatorname{Re}_{\mathrm{r}}=\Omega \mathrm{D}^{2} \rho /(2 \mu)$, where $\mathrm{V}$ is the volumetric flow rate, $\rho$ and $\mu$ are respectively the fluid density and dynamic viscosity, $D$ is the inner diameter of the shaft and $\omega$ is the rotational speed. The Nusselt number is given by (1) and (2), with the convection coefficient $h=\mathrm{Nu}_{D} \mathrm{k} / \mathrm{D}$.

$$
\begin{aligned}
& N u_{D}=0.01963 R e_{a}^{0.9285}+8.5101 \times 10^{-6} R e_{r}^{1.4513} \\
& \quad \text { for } R e_{a}<3 \times 10^{4} ; 1.6 \times 10^{3}<R e_{r} \leq 2.77 \times 10^{5} \\
& N u_{D}=2.85 \times 10^{-4} R e_{r}^{1.19} \text { for } R e_{r}>2.77 \times 10^{5}
\end{aligned}
$$

Based on the work of Celik et al. [13], the modeling of a RHP can be approximated by the equivalent thermal network in Fig. 6. The most important resistances will be discussed separately. The heat source can be seen as a fraction of the losses dissipated in the rotor. Resistances $\mathrm{R}_{1}, \mathrm{R}_{2}, \mathrm{R}_{6}$ and $\mathrm{R}_{8}$ are only dependent on their corresponding conductive heat flow path. These depend on material properties and specific geometries. Hence, these are not discussed in detail. The thermal resistance resulting from the vapor pressure drop $\left(\mathrm{R}_{4}\right)$

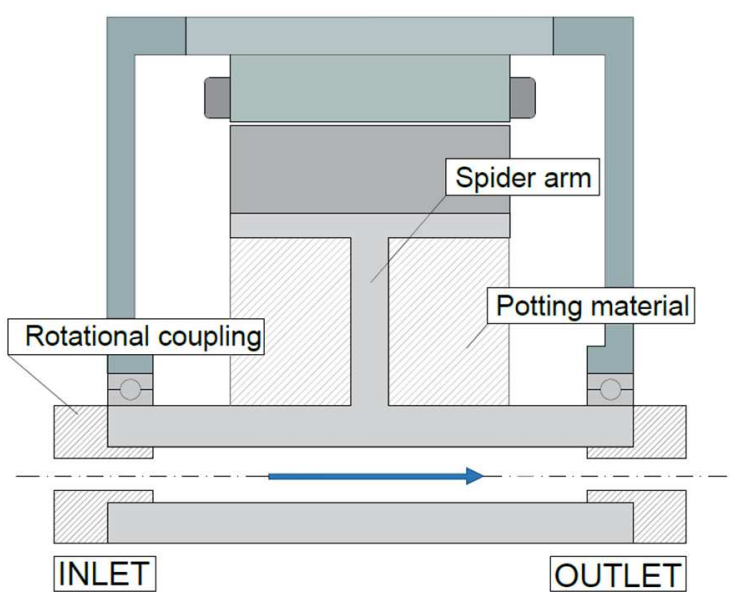

Fig. 3. Hollow shaft liquid cooling

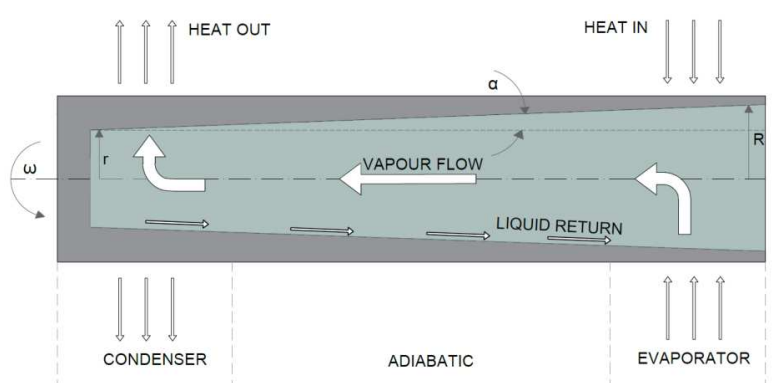

Fig. 4. Rotating heat pipe cooling working principle

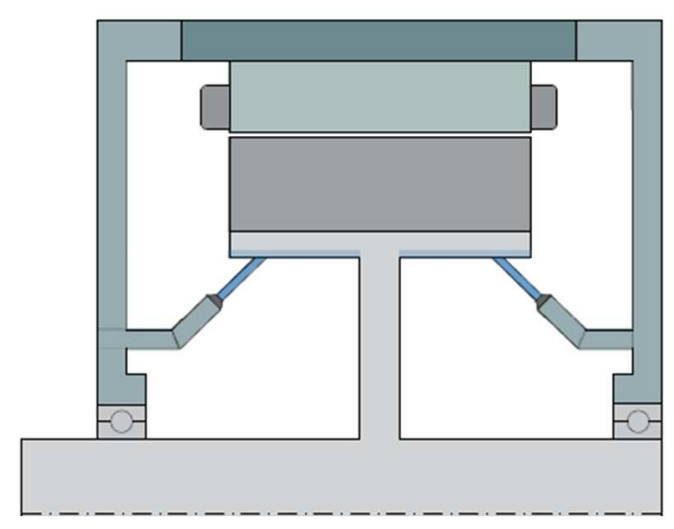

Fig. 5. Rotor jet cooling schematic

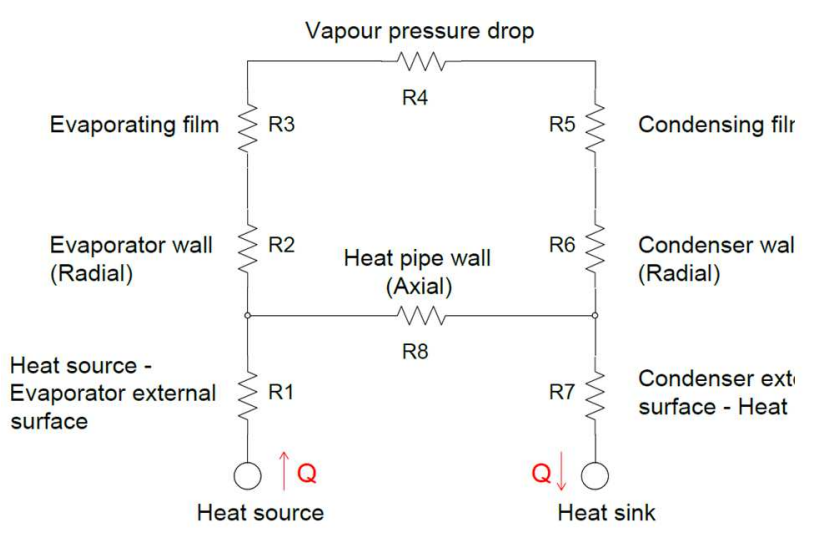

Fig. 6. Rotating heat pipe cooling schematic 
can be neglected since it is small [13]. The thermal resistance between the condenser external surface $\mathrm{R}_{7}$ and the heat sink will depend on the flow and heat sink properties. In this work, the thermal resistance at the evaporator side of the RHP $\left(\mathrm{R}_{3}\right)$ is determined based on the Nusselt film theory. Song et al. [14] argued that for moderate-speed rotating heat pipes (2000-4000 $\mathrm{rpm}$ ), the heat transfer coefficient $\mathrm{h}_{\text {evaporation }}=\mathrm{Nu}_{\mathrm{D}} \mathrm{k} / \delta$ at the evaporator side can be approximated by a correlation for natural convection in liquid pools under acceleration, when the liquid film Rayleigh number $\mathrm{Ra}_{\delta}=\rho_{1} \Omega^{2} \mathrm{D} \beta \Delta \mathrm{T} \delta^{3} /\left(2 \mu_{1} \alpha_{1}\right)$ is high enough. The Nusselt number correlation is given by $\mathrm{Nu}_{\delta}=0.133 \mathrm{Ra}_{\delta}{ }_{\delta}^{0.375}$ for $400 \leq \mathrm{Ra}_{\delta}$ and otherwise $\mathrm{Nu}_{\delta}=1$, where $\alpha_{1}$ and $\beta_{1}$ are respectively the thermal diffusivity and thermal expansion coefficient of the liquid. D is the maximum RHP inner diameter and $\delta$ is the liquid film thickness. In this work, the latter variable is assumed to be $\delta=5 \times 10^{-4} \mathrm{~m}$ [13]. The temperature difference $\Delta \mathrm{T}$ is the difference between the wall temperature and the bulk temperature. The condensing heat transfer coefficient and hence thermal resistance of the condensing film $\left(\mathrm{R}_{5}\right)$ is defined by the correlation proposed by Shukla et al. [15], which has two drawbacks. A first one is that the correlation is developed for water as a working fluid. This means that for the remaining RHP research, the working fluid is limited to water. The second drawback is that the correlation holds for rotational velocities ranging from $1000 \mathrm{rpm}$ to 2000 $\mathrm{rpm}$. This means that for high rotational speeds the correlation will not be completely reliable. The correlation for the heat transfer coefficient by condensation is given by $h_{\text {cond }}=$ $440 Q^{0.1} \mathrm{Fr}^{0.3}$, where $\mathrm{Fr}=\mathrm{D} \Omega^{2} /(2 \mathrm{~g})$ is the Froude number, $\mathrm{Q}$ is the heat transfer rate and $g$ is the gravitational acceleration.

In rotor jet cooling, the cooling is realized by impinging liquid jets on the rotor and subsequent flow of the fluid over the rotor surfaces. For simplicity, heat is considered as only removed in the immediate vicinity of the jets. A correlation for the local heat transfer resulting from static impinging pipe nozzle jets is proposed by Ma et al. [16] [17]: $N u_{r}=$ $N u_{0} \frac{\left(1.85 \times 10^{-3} R e_{d}\right)^{5.82 \times 10^{-2}(r / d)}}{1+0.236(r / d)^{1.9}}$ with $\mathrm{Nu}_{0}=1.27 \operatorname{Re}_{\mathrm{d}}{ }^{0.495} \operatorname{Pr}^{1 / 3}$. In this equation, $d$ is the nozzle diameter, $\mathrm{Re}_{\mathrm{d}}$ is the Reynolds number based on the nozzle exit conditions, Pr is the Prandtl number and $\mathrm{r}$ is the distance relative to the center impingement point. The correlation is valid in the circular range limited by 10 times the nozzle diameter as radius. As the correlation predicts local Nusselt numbers, an averaged global Nusselt number was calculated based on the amount of jets, spider arm inner perimeter and location of the different nodes to the center impingement point. Within the tangential direction, the convection coefficients are uniformly distributed over the inner surface of the spider arm, which is justified since the jets are static (mounted within the end plates) and impinging on a rotating surface. Within the axial direction, the distance from the jet center impingement point is taken into account when calculating the average convection coefficient for the different nodes.

Some additional modeling considerations include the heat transfer through the air gap, heat transfer in the end space and the modeling of the windings. The air gap convection coefficient, and consequently the heat transfer through the air gap is estimated by the correlation of Becker and Kaye [18]. The windings consist of rectangular conductors with appropriate insulation material, surrounded by impregnation material. Equivalent anisotropic thermal conductivities were determined, based on the geometry and thermal conductivities of the different materials.

\section{RESULS AND DISCUSSION}

The evaluation of the different rotor cooling methods is made, based on the temperature distribution in the machine and maximum coil and permanent magnet temperatures. All temperatures are shown relative to the temperature of the coolant with which the electric machine is cooled. For the simulations of the jacket cooling, a $60 / 40 \%$ by mass mixture of water-glycol (WG) and flow rate of $12 \mathrm{l} / \mathrm{min}$ are used. For the hollow shaft liquid cooling the same settings as the jacket are used with a remaining shaft wall thickness of $16 \mathrm{~mm}$. The remaining shaft thickness is the same for the rotating heat pipe with water as the working fluid. The condenser side of the rotating heat pipe is assumed to be $16 \mathrm{~mm}$ long and at the temperature of the coolant. In order to maximize the performance of rotor jet cooling, it was observed that for a fixed flow rate of $5 \mathrm{l} / \mathrm{min}$ the amount of nozzles and the nozzle diameter should be maximized. Due to practical reasons, 24 nozzles with a diameter of $2.5 \mathrm{~mm}$ are used, spraying Automatic Transmission Fluid on the inner surface of the spider arm at a temperature of $20^{\circ} \mathrm{C}$ higher than the $\mathrm{WG}$ of the jacket. As the rotor cooling techniques are not sufficient to cool the electrical machine autonomously, they are complementary used with conventional jacket cooling.

Fig. 7 shows the temperature distribution in different sections of the front side of the electric machine for reference jacket cooling case in the high speed operating point: the end plate (left), end winding (second) and four cross-sections of the active region at equidistant axial positions (last) are shown. For this case, the maximum relative coil temperature is $90.4^{\circ} \mathrm{C}$. As the maximum relative PM temperature reaches $123.7^{\circ} \mathrm{C}$, additional rotor cooling techniques will be necessary.

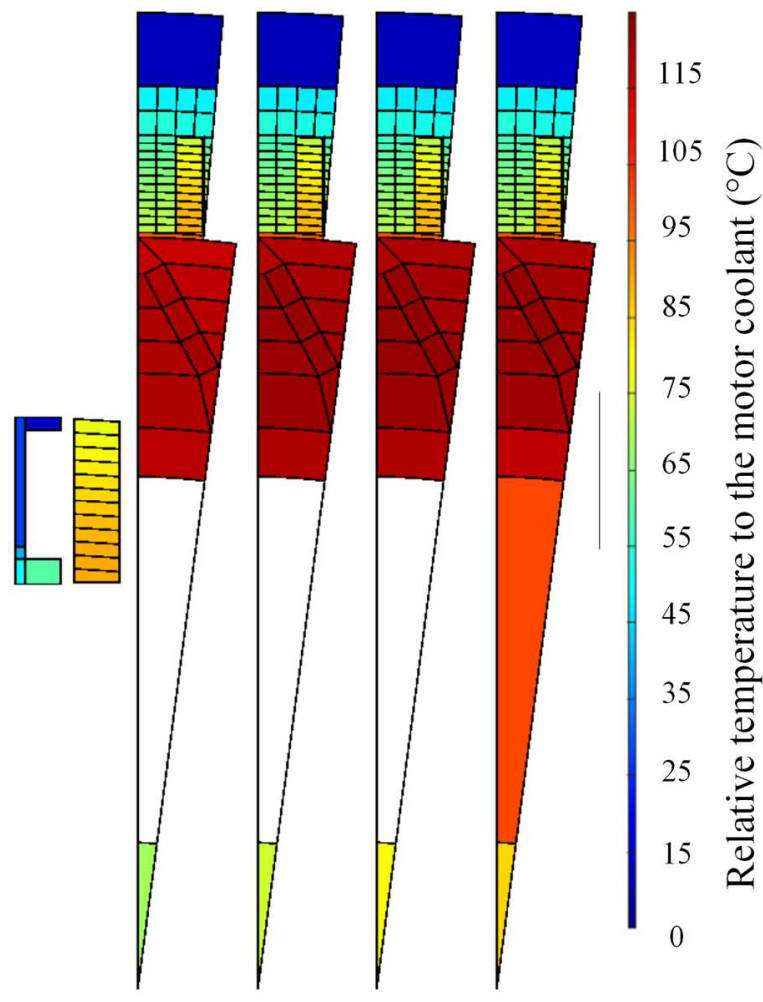

Fig. 7. Reference water jacket cooling 
When additionally to the water jacket cooling, HSLC is implemented, the maximum relative PM temperature reduces almost with $20^{\circ} \mathrm{C}$ to $104.9^{\circ} \mathrm{C}$. The reduction in maximum coil temperatures is less pronounced: a decrease of $6.1^{\circ} \mathrm{C}$ is observed, resulting in a maximum relative coil temperature of $84.3^{\circ} \mathrm{C}$. The rotational Reynolds number is rather high at the high speed operating point such that (2) should be used to calculate the mean convection coefficient at the hollow shaft wall which equals $11162 \mathrm{~W} / \mathrm{m}^{2} \mathrm{~K}$. The resulting temperature distribution plot is shown in Fig. 7. For the rotating heat pipe cooling, the evaporator side of the RHP is taken as the total machine length. This means that next to the cooling of the active part, also the bearings are directly cooled. The calculated mean convection coefficient of the rotating heat pipe equals $6578 \mathrm{~W} / \mathrm{m}^{2} \mathrm{~K}$ at the evaporator side and 6031 $\mathrm{W} / \mathrm{m}^{2} \mathrm{~K}$ at the condenser side. When comparing the results of HSLC and RHP cooling (shown in Fig. 9), similar temperature distributions are seen. However, the maximum temperatures are slightly higher for the RHP cooling. The PM temperatures are the highest, reaching a maximum relative value of $107.7^{\circ} \mathrm{C}$. The maximum relative coil temperature is $85.3^{\circ} \mathrm{C}$.

It is observed that the condenser area is rather small compared to the evaporator area of the rotating heat pipe. A sensitivity analysis showed that doubling the condenser area only results in a temperature decrease in the permanent magnets of $1.2^{\circ} \mathrm{C}$. These results show that both HSLC and RHP cooling are not effective due to the thermal bottleneck between the shaft and the rotor iron. Simulations of HSLC with an increased thermal conductivity of the potting material to $3.5 \mathrm{~W} / \mathrm{mK}$ shows a reduction in the maximum rotor temperature of $23.7^{\circ} \mathrm{C}$ with respect to the reference case or an additional temperature reduction of $4.9^{\circ} \mathrm{C}$ in the permanent magnets compared to the case with a potting of $2.3 \mathrm{~W} / \mathrm{mK}$. In this case, $46.6 \%$ of the heat generated in the rotor is transferred to the shaft, whereas this percentage is only $15.7 \%$ if no potting material would be used. For the rotating heat pipe cooling, similar effects were observed.

In case of rotor jet cooling, the calculated mean convection coefficient over the whole inner surface of the spider arm equals $385 \mathrm{~W} / \mathrm{m}^{2} \mathrm{~K}$. The mean convection coefficient is rather small compared to the other cooling methods since it uses oil as heat transfer fluid which has worse thermal properties compared to water-glycol, but also because only several jets are pointing on a much larger surface area compared to the other methods. For the rotor jet cooling method, the permanent magnets reach a maximum relative temperature of $70.8^{\circ} \mathrm{C}$. As such, when using rotor jet cooling, the temperature reduces with more than $50^{\circ} \mathrm{C}$ with respect to the reference case. The overall maximal relative temperature is now found in the coils and is equal to $76.7^{\circ} \mathrm{C}$. Rotor jet cooling thus results in a temperature decrease of $13.7^{\circ} \mathrm{C}$ in the coils with respect to the reference case, while the other rotor cooling techniques only result in a decrease of about $5^{\circ} \mathrm{C}$. In this case, less heat is transferred from the rotor to the stator through the air gap, resulting in lower stator and coil temperatures. In Fig. 7 (d), it is seen that the electrical motor tends to be more isothermal and no explicit hot spots are observed. The thermal bottleneck that causes the shaft cooling techniques to be ineffective, is not present when applying the rotor jet cooling technique. As the oil impinges on the spider arm steel, forced convection takes place near the rotor iron, which has a good radial thermal conductivity.

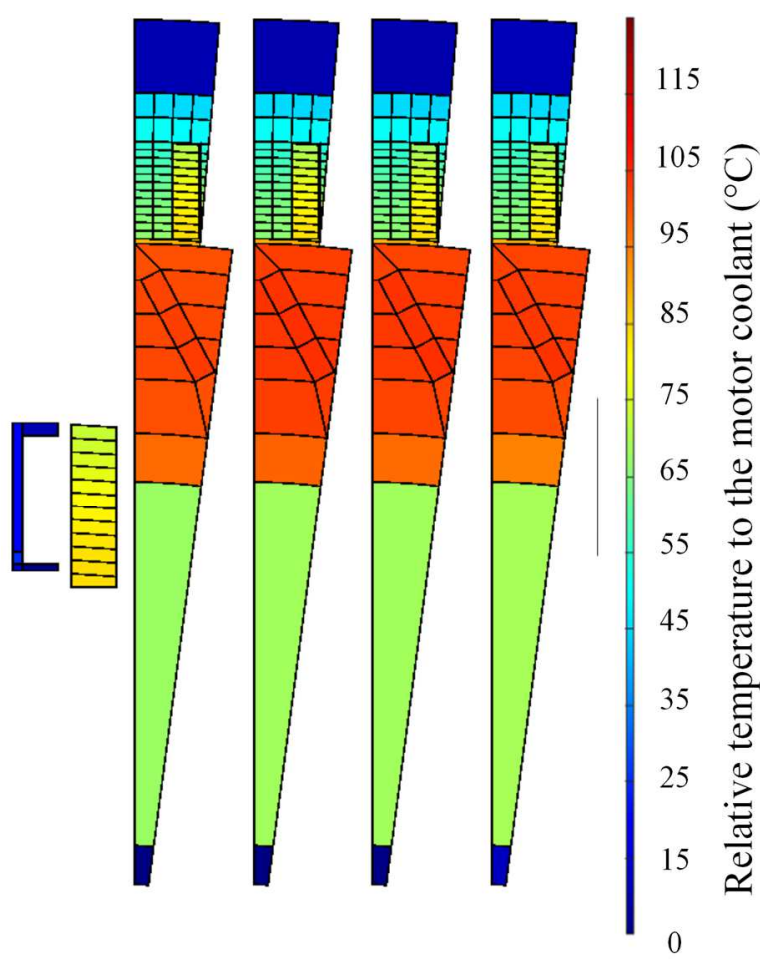

Fig. 8. Water jacket combined with hollow shaft liquid cooling

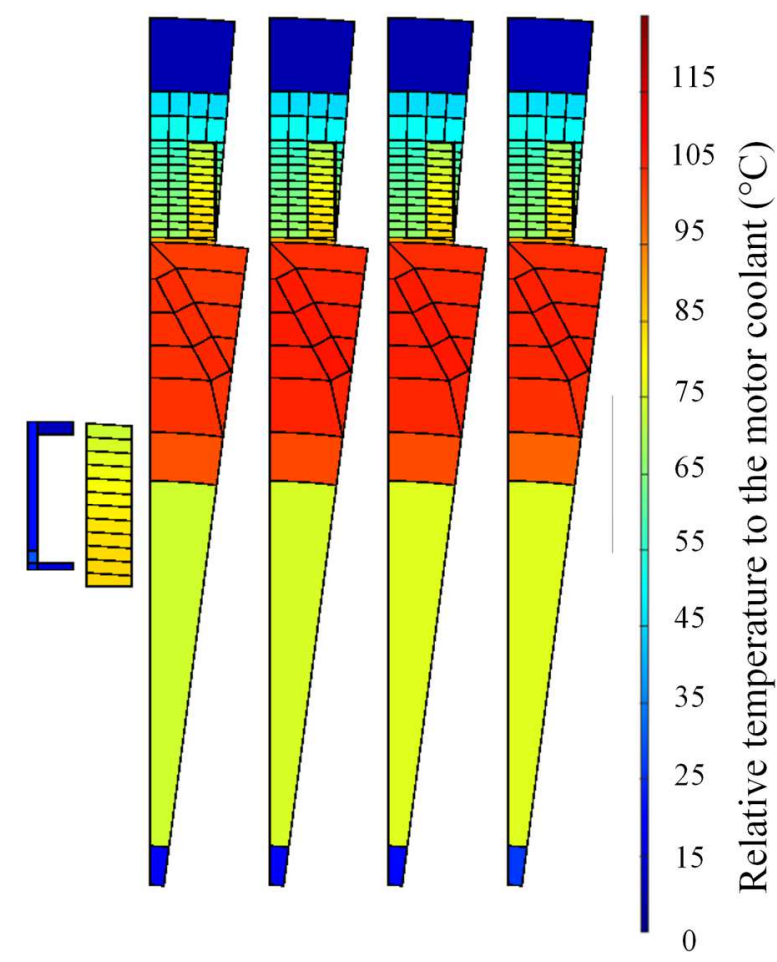

Fig. 9. Water jacket combined with rotating heat pipe cooling 


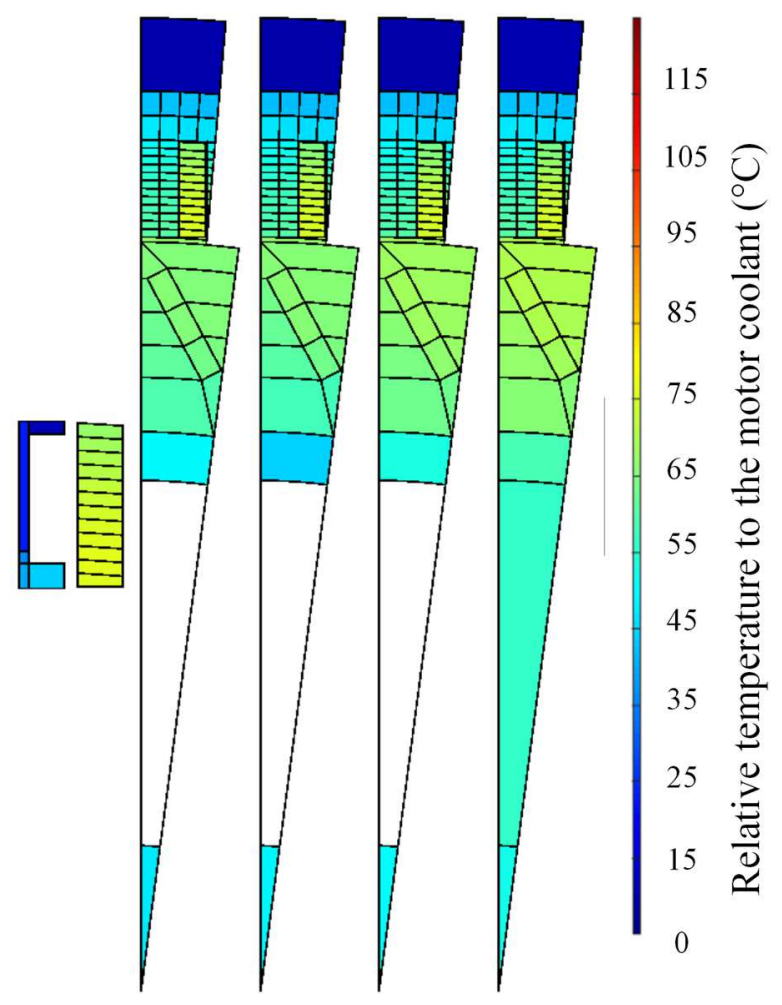

Fig. 10. Water jacket combined with rotor jet cooling

\section{CONCLUSION}

Different rotor cooling methods were modeled using an existing LPM and the thermal performance of several rotor cooling methods was investigated based on an existing jacket cooled IPMSM. Hollow shaft liquid cooling and rotating heat pipe cooling result in equivalent temperature distributions in the motor components. The full potential of these cooling methods is not utilized due to the specific geometry of the considered electrical machine. As the rotor iron is carried by a steel structure, a thermal bottleneck is introduced. It is concluded that for these cooling techniques the actual cooling is located unfavorable with respect to the locations where the losses are generated. The most effective cooling technique is rotor jet cooling, as forced convection takes place directly on the spider arm steel near the rotor iron.

\section{ACKNOWLEDGMENT}

This research was supported by Flanders Make, the strategic research center for the manufacturing industry, and the HERMESFONDS in the framework of the SingleOilCnL project (HBC.2020.2009).

\section{REFERENCES}

[1] L. Zhiyong, W. Xuehuan, and C. Linhong, "Thermal analysis of PMSM based on lumped parameter thermal network method," in 2016 19th International Conference on Electrical Machines and Systems (ICEMS), 2016, pp. 1-5.

[2] Y. Yang, B. Bilgin, M. Kasprzak, S. Nalakath, H. Sadek, M. Preindl, et al., "Thermal management of electric machines," IET Electrical Systems in Transportation, vol. 7, pp. 104-116, 2016.
[3] D. Tanguy, S. HARMAND, J. PELLE, and R. YU, "Experimental study of oil cooling systems for electric motors," Applied thermal engineering, vol. 71, 2014.

[4] A. Carriero, M. Locatelli, K. Ramakrishnan, G. Mastinu, and M. Gobbi, "A Review of the State of the Art of Electric Traction Motors Cooling Techniques," 2018.

[5] M. Schiefer and M. Doppelbauer, "Indirect slot cooling for high-power-density machines with concentrated winding," in 2015 IEEE International Electric Machines \& Drives Conference (IEMDC), 2015, pp. 1820-1825.

[6] Y. Gai, M. Kimiabeigi, Y. C. Chong, J. D. Widmer, X. Deng, M. Popescu, et al., "Cooling of automotive traction motors: schemes, examples, and computation methods," IEEE Transactions on Industrial Electronics, vol. 66, pp. 1681-1692, 2018.

[7] J. Nonneman, I. T'Jollyn, P. Sergeant, and M. De Paepe, "Quality Assessment of a 2D FE Based Lumped Parameter Electric Motor Thermal Model Using 3D FE Models," in 2020 International Conference on Electrical Machines (ICEM), 2020, pp. 973-980.

[8] Y. Gai, M. Kimiabeigi, Y. C. Chong, J. D. Widmer, J. Goss, U. SanAndres, et al., "On the measurement and modeling of the heat transfer coefficient of a hollow-shaft rotary cooling system for a traction motor," IEEE Transactions on Industry Applications, vol. 54, pp. 5978-5987, 2018.

[9] W. Lian and T. Han, "Flow and heat transfer in a rotating heat pipe with a conical condenser," International Communications in Heat and Mass Transfer, vol. 101, pp. 70-75, 2019.

[10] D. H. Lim and S. C. Kim, "Thermal performance of oil spray cooling system for in-wheel motor in electric vehicles," Applied Thermal Engineering, vol. 63, pp. 577-587, 2014.

[11] Q. Lu, R. Muthukumar, H. Ge, and S. Parameswaran, "Numerical study of a rotating liquid jet impingement cooling system," International Journal of Heat and Mass Transfer, vol. 163, p. 120446, 2020.

[12] S. Seghir-Ouali, D. Saury, S. Harmand, O. Phillipart, and D. Laloy, "Convective heat transfer inside a rotating cylinder with an axial air flow," International journal of thermal sciences, vol. 45, pp. 1166-1178, 2006.

[13] M. Celik, G. Paulussen, D. Van Erp, W. De Jong, and B. J. Boersma, "Transient modelling of rotating and stationary cylindrical heat pipes: An engineering model," Energies, vol. 11, p. 3458, 2018.

[14] F. Song, D. Ewing, and C. Ching, "Heat transfer in the evaporator section of moderate-speed rotating heat pipes," International journal of heat and mass transfer, vol. 51, pp. 1542-1550, 2008.

[15] K. Shukla, A. B. Solomon, and B. Pillai, "Experimental studies of rotating heat pipes," Heat Transfer-Asian Research: Co - sponsored by the 
Society of Chemical Engineers of Japan and the Heat Transfer Division of ASME, vol. 38, pp. 475484, 2009.

[16] C. Ma, Q. Zheng, and S. Ko, "Local heat transfer and recovery factor with impinging free-surface circular jets of transformer oil," International journal of heat and mass transfer, vol. 40, pp. 4295-4308, 1997.

[17] X. Feng, E. Cousineau, K. Bennion, G. Moreno, B. Kekelia, and S. Narumanchi, "Experimental and numerical study of heat transfer characteristics of single-phase free-surface fan jet impingement with automatic transmission fluid," International Journal of Heat and Mass Transfer, vol. 166, p. 120731, 2021.

[18] D. A. Howey, P. R. Childs, and A. S. Holmes, "Air-gap convection in rotating electrical machines," IEEE transactions on industrial electronics, vol. 59, pp. 1367-1375, 2010. 